\title{
Antenatal care visit attendance, intermittent preventive treatment during pregnancy (IPTp) and malaria parasitaemia at delivery
}

Judith K Anchang-Kimbi ${ }^{{ }^{*}}$, Eric A Achidi ${ }^{2}$, Tobias O Apinjoh ${ }^{2}$, Regina N Mugri ${ }^{3}$, Hanesh Fru Chi ${ }^{3}$, Rolland B Tata ${ }^{3}$, Blaise Nkegoum ${ }^{4}$, Joseph-Marie N Mendimi ${ }^{4}$, Eva Sverremark-Ekström ${ }^{5}$ and Marita Troye-Blomberg ${ }^{5}$

\begin{abstract}
Background: The determinants and barriers for delivery and uptake of IPTp vary with different regions in sub-Saharan Africa. This study evaluated the determinants of ANC clinic attendance and IPTp-SP uptake among parturient women from Mount Cameroon Area and hypothesized that time of first ANC clinic attendance could influence uptake of IPTp-SP/dosage and consequently malaria parasite infection status at delivery.

Methods: Two cross sectional surveys were carried out at the Government Medical Centre in the Mutengene Health Area, Mt Cameroon Area from March to October 2007 and June 2008 to April 2009. Consented parturient women were consecutively enrolled in both surveys. In 2007, socio-demographic data, ANC clinic attendance, gestational age, fever history and reported use/dosage of IPTP-SP were documented using a structured questionnaire. In the second survey only IPT-SP usage/dosage was recorded. Malaria parasitaemia at delivery was determined by blood smear microscopy and placental histology.
\end{abstract}

Results and discussion: In 2007, among the 287 women interviewed, 2.2\%, 59.7\%, and 38.1\% enrolled in the first, second and third trimester respectively. About $90 \%$ of women received at least one dose SP but only $53 \%$ received the two doses in 2007 and by 2009 IPTp-two doses coverage increased to 64\%. Early clinic attendance was associated $(\mathrm{P}=0.016)$ with fever history while being unmarried $(\mathrm{OR}=2.2 ; 95 \% \mathrm{Cl}: 1.3-3.8)$ was significantly associated with fewer clinic visits (<4visits). Women who received one SP dose ( $\mathrm{OR}=3.7 ; 95 \% \mathrm{Cl}$ : 2.0-6.8) were more likely not to have attended $\geq 4$ visits. A higher proportion $(P<0.001$ ) of women with first visit during the third trimester received only one dose, meanwhile, those who had an early first ANC attendance were more likely $(\mathrm{OR}=0.4 ; 95 \% \mathrm{Cl}=0.2-0.7)$ to receive two or more doses. Microscopic parasitaemia at delivery was frequent $(P=0.007)$ among women who enrolled in the third trimester and had received only one SP dose than in those with two doses.

Conclusion: In the study area, late first ANC clinic enrolment and fewer clinic visits may prevent the uptake of two SP doses and education on early and regular ANC clinic visits can increase IPTp coverage.

Keywords: Antenatal care visit attendance, IPTp, Malaria parasite infection

\footnotetext{
* Correspondence: kuoh2000@yahoo.fr

'Department of Zoology and Animal Physiology, University of Buea, Buea 63, Cameroon

Full list of author information is available at the end of the article
}

\section{Biomed Central}

(c) 2014 Anchang-Kimbi et al.; licensee BioMed Central Ltd. This is an Open Access article distributed under the terms of the Creative Commons Attribution License (http://creativecommons.org/licenses/by/2.0), which permits unrestricted use, distribution, and reproduction in any medium, provided the original work is properly credited. The Creative Commons Public Domain Dedication waiver (http://creativecommons.org/publicdomain/zero/1.0/) applies to the data made available in this article, unless otherwise stated. 


\section{Background}

Antenatal care is an important component of maternal and child health care to monitor the health of the mother, anticipate difficulties and complications of labour, ensure the birth of a healthy baby and to help the mother rear the child [1]. Infrequent, late or non-attendance at antenatal care clinics has been associated with adverse maternal outcome [2]. Malaria in pregnancy remains a major public health problem in endemic areas of the African region, where approximately 25-30 million of pregnant women are at risk of Plasmodium falciparum infection and its adverse outcomes during their pregnancy $[3,4]$. To reduce malaria burden in pregnancy, the World Health Organization (WHO) currently recommends a package of interventions in areas with stable (high) transmission of P. falciparum [4]. These include the use of insecticide-treated nets (ITNs), intermittent preventive treatment (IPT) and effective case management of malaria illness and anaemia. Currently, sulphadoxine-pyrimethamine (SP) is the most effective drug for IPT despite the increasing resistance of SP to $P$. falciparum $[5,6]$. Intermittent preventive therapy with SP for malaria in pregnancy (IPTp-SP) in areas of high or seasonal transmission have been shown to lower placental infection rates, increase both maternal haemoglobin levels and the infants' birth weight $[7,8]$.

A high proportion of pregnant women in Africa attend ANC clinic at least once during pregnancy thus the antenatal clinic provides a good opportunity for delivering interventions to control malaria in pregnancy in endemic regions. Studies conducted in Malawi and Kenya showed that the maximum benefit of IPT can be gained by receiving two doses or more (in HIV infected pregnant women) doses of SP. More so, majority of women who received a minimum of two doses of SP do attend four or more ANC visits [9]. Despite a high ANC clinic attendance in most countries in Sub-Africa, reported coverage level of IPTp-SP and ITNs remains below the reversed Abuja Declaration target of $80 \%$ [10].

The determinants and barriers for delivery and uptake of IPT and ITN vary with different regions [11]. Metaanalysis of factors affecting the delivery, access, and use of interventions to prevent malaria in pregnancy in subSaharan Africa identified education, knowledge and perception about malaria [12,13], socio-economic status [14-17], number and timing of antenatal clinic visits [12,17], number of pregnancies, healthcare system issues $[13,17]$ as factors that influence intervention coverage as well as access to ANC services. While most reports on ANC clinic attendance and IPTp/SP coverage are from studies carried out in West and East Africa, reports from Central Africa are limited [17].

In 2007, about 90\% of women in Mount Cameroon Area attending ANC clinic received IPTp-SP at least once during pregnancy, but only $52 \%$ of these women had the recommended two or more doses [18]. To identify facilitating factors for scaling up IPTp-SP coverage in the study area, this study evaluated the determinants of ANC clinic attendance and IPTp-SP uptake among parturient women who seek delivery services at the Mutengene Medical Centre. The study hypothesized that time of first ANC clinic attendance could influence uptake of IPTp-SP/ dosage and consequently malaria parasite infection status among pregnant women at delivery.

\section{Methods}

\section{Study area and population}

This study consists of two cross sectional surveys carried out at the Government Medical Centre in the Mutengene Health Area, Mt Cameroon Area, Southwest Region from March to October 2007 and June 2008 to April 2009. The Mutengene Medical Centre is the only government owned institution that offers antenatal care, preventive and curative services at subsidized costs in the health area. The characteristics of the study area have been described elsewhere [18]. In brief, malaria transmission is perennial, with some seasonality. Pregnant women in this semi-urban area of Cameroon are frequently (62\%) exposed to $P$. falciparum infection during pregnancy with placental malaria parasitaemia rate of $34 \%$ at delivery. History of fever attack is common (44\%) in women during pregnancy [18]. Previous studies show that anaemia is still a major health problem in this area [18-20]. Consecutive pregnant women reporting for delivery at the Mutengene maternity and who consented to participate in the study were enrolled.

\section{Administration of questionnaires}

During the first survey, a total of 287 parturient women were interviewed. A structured questionnaire was administered to women to document socio-demographic data including age, residence, marital status and educational level. Antenatal data were obtained from either the antenatal card or the register of the centre with assistance of a midwife and included: parity, gestational age at first ANC, number of ANC visits made. Gestational age was determined after the physical examination by the midwife who used a gestational calendar or uterine growth when the date of last menses was not known. Fever history, use of IPTpSP (dosage and dates of drug administration) was recorded from ANC cards, patient's medical record book and health centre maternal care register. During the second survey, only IPTp-SP usage/dosage was recorded for 292 pregnant women at $\geq 36$ weeks of gestation.

\section{Sample collection and processing}

Maternal peripheral venous blood $(2 \mathrm{ml})$ was collected within 24 hours after delivery for malaria parasite detection. Immediately following delivery, the placenta was obtained and a small piece of placental tissue $\left(0.5 \mathrm{~cm}^{3}\right)$ 
excised from the centre of the placenta to prepare impression smears. A larger $\left(4 \mathrm{~cm}^{3}\right)$ biopsy specimen of placental tissue was fixed in $10 \%$ neutral buffered formalin for histopathological assessment as described elsewhere [18].

\section{Parasitological examination}

Peripheral blood was used to prepare thick blood smears and together with placental impression smears stained with Giemsa (Sigma) and examined by light microscopy. Placental tissue sections were processed, stained with haematoxylin and eosin and examined. Placental malaria parasitisation was determined as reported elsewhere [18].

\section{Definitions and data analysis}

All data were entered and analysed using SPSS version 19 . Age and parity was categorized as follows: age $(\leq 20$, $21-25,>25$ ) years; parity (primiparae, secundiparae and multiparae ( $\geq 3$ pregnancies). The Complete ANC attendance was considered to be at least four ANC visits during pregnancy. A full course IPTp/SP was defined as at least a complete two-dose course of SP administered during the pregnancy. Malaria infection was defined as the presence of parasites detected by peripheral blood, placental blood microscopy and placental histologic examination further classified as microscopic and submicroscopic infection.

Differences between groups were assessed using chisquare or Fisher's exact tests for proportions and student $t$-test for means. The factors that influence ANC clinic attendance and IPTp-SP uptake were assessed using odds ratio and the 95\% confidence interval (OR (95\% CI) determined in univariate analysis and logistic regression in multivariate analysis. Correlations were assessed using Pearson correlation test. A p-value of less than 0.05 was considered significant. The study received ethical clearance from the Delegation of Public Health Ethics Review Board, Buea, Southwest Region.

\section{Results}

\section{Characteristics of study participants}

The characteristics of parturient women who participated in the study in 2007 are shown in (Table 1). Their ages range from 14-40 years with a median age and parity of 23(20-27) years and 2(1-3) births respectively. There was strong positive correlation $(\mathrm{r}=0.796 ; \mathrm{P}<0.001)$ between age and parity of all mothers, where primiparous mothers $(19.6 \pm 2.7$ years) were significantly younger $(\mathrm{p}<$ $0.001)$ compared to multiparous mothers (25.8 \pm 4.7 years). The study population was predominantly made up of married women $(75.1 \%)$; and $62.3 \%$ of the women were educated to primary level and only $4.5 \%$ and $6.4 \%$ had tertiary level and no formal education respectively. It is noted that $59.2 \%(125 / 211)$ of the married women were housewives and engaged in no occupation while $52.2 \%$ (35/67) of the
Table 1 Socio-demographic characteristics of participants $(\mathrm{N}=\mathbf{2 8 7})$

\begin{tabular}{lc}
\hline Factor & \% (n) \\
\hline Age (years) & \\
$\leq 20$ & $30.3(86)$ \\
$21-25$ & $35.9(102)$ \\
$>25$ & $33.8(96)$ \\
Parity & \\
Primiparous & $30.7(88)$ \\
Secundiparous & $28.9(83)$ \\
Multiparous & $40.4(116)$ \\
Educational level & \\
None & $6.4(17)$ \\
Primary & $62.3(165)$ \\
Secondary & $26.8(71)$ \\
Tertiary & $4.5(12)$ \\
Marital status & \\
Married & $75.1(214)$ \\
Unmarried & $24.9(71)$ \\
Occupation & \\
Housewife & \\
Student & $45.9(128)$ \\
Business & $16.5(46)$ \\
Farming & $30.8(86)$ \\
Profession & $5.4(15)$ \\
\hline & $1.4(4)$ \\
\hline
\end{tabular}

unmarried mothers were students or applicants. At delivery, the median gestational age of 39 (38-40) weeks and neonatal birth weight of $3.20(3.00-3.60) \mathrm{kg}$ respectively were recorded.

\section{Antenatal care attendance}

Of the 287 participating women, 96.5\% reported attending $\mathrm{ANC}$ at least once during their last recent pregnancy. Antenatal clinic visits varied from 1-12 with a median of 5 visits. Among ANC attendees, majority (60\%) made their first visit in the second trimester (Table 2). Approximately $91 \%$ of the women made more than one visit and only $69.3 \%$ of the ANC attendees had complete attendance (considered to be at least four ANC visits during pregnancy) (Table 2). Women who had a complete ANC attendance were significantly older $(\mathrm{P}=0.038)$ when compared with those who did fewer ANC visits. Also married women frequently made four or more visits than unmarried women. Thus younger age ( $\leq 20$ years) $(\mathrm{OR}=2.0 ; 95 \% \mathrm{CI}: 1.2-3.4)$ and being unmarried $(\mathrm{OR}=2.2$; $95 \% \mathrm{CI}: 1.3-3.8)$ were significant risk factors associated with fewer clinic visits (<4visits). Fever history was associated with more ANC clinic visits (OR $=0.51,95 \%$ CI 0.30 - 0.89) (Table 3) as women with fever history 
Table 2 Antenatal care visit attendance and IPTp-SP usage of parturient women $(\mathrm{N}=\mathbf{2 8 7})$

\begin{tabular}{|c|c|c|}
\hline Characteristics & \multicolumn{2}{|c|}{$\%(n)$} \\
\hline ANC visit at least once & \multicolumn{2}{|c|}{$96.5(277)$} \\
\hline ANC visit at least twice & \multicolumn{2}{|c|}{$94.2(261)$} \\
\hline ANC visit at least thrice & \multicolumn{2}{|c|}{$84.1(233)$} \\
\hline$\geq 4$ ANC visits & \multicolumn{2}{|c|}{$71.8(199)$} \\
\hline Median No. of ANC visits (quartiles) & \multicolumn{2}{|c|}{$5(3-6)$} \\
\hline Non-ANC attendance & \multicolumn{2}{|c|}{$3.5(10)$} \\
\hline \multicolumn{3}{|l|}{ First ANC visit; Median GA (range) } \\
\hline $1^{\text {st }}$ trimester & $2.2(06)$ & $12(8-12)$ \\
\hline $2^{\text {nd }}$ trimester & $59.7(160)$ & $21(14-25)$ \\
\hline $3^{\text {rd }}$ trimester & $38.1(102)$ & $29(26-37)$ \\
\hline IPTp-SP usage & \multicolumn{2}{|c|}{$90.5(258 / 285)$} \\
\hline \multicolumn{3}{|l|}{ SP dosage } \\
\hline Two or more & \multicolumn{2}{|c|}{$53.1(136)$} \\
\hline One & \multicolumn{2}{|c|}{$46.9(120)$} \\
\hline No IPTp-SP & \multicolumn{2}{|c|}{$9.5(27)$} \\
\hline \multicolumn{3}{|l|}{ Trimester of $1^{\text {st }}$ SP dose } \\
\hline $1^{\text {st }}$ trimester & \multicolumn{2}{|c|}{$0.8(2)$} \\
\hline $2^{\text {nd }}$ trimester & \multicolumn{2}{|c|}{$59.8(150)$} \\
\hline $3^{\text {rd }}$ trimester & \multicolumn{2}{|c|}{$39.4(99)$} \\
\hline
\end{tabular}

$\mathrm{GA}=$ Gestational age (weeks).

$(22.13 \pm 4.73$ weeks $)$ reported earlier $(\mathrm{t}=-4.790 ; \mathrm{P}<0.001)$ for first ANC clinic visit when compared with those with no fever history $(25.00 \pm 4.80$ weeks $)$. In multivariate analysis, marital status (unmarried) $(\mathrm{p}=0.006)$ and fever history $(\mathrm{P}=0.009)$ remained significant independent factors associated with ANC visit attendance (Table 3).

To further investigate the timeliness of ANC attendance, a categorization of 'early first attendance' was defined as a first visit to ANC at or before 4 months gestation. Of all respondents only $18.7(50 / 268)$ had attended ANC at or before four months of gestation. Women with a fever history were likely $(60.0 \%$ vs $40.0 \%$; $\left.\chi^{2}=5.841 ; \mathrm{P}=0.016\right)$ to make an early first $\mathrm{ANC}$ visit than those without fever history. No other determinants of early attendance were identified.

\section{IPTp uptake at ANC clinic}

Sulphadoxine-pyremathmine treatment was presumptive and administered at an ANC. Among ANC attendees, 90.5\% reported receiving at least one dose of SP during pregnancy. However, only $53.1 \%(136 / 256)$ received two or more doses of IPT-SP at an ANC visit. Among women who received SP at least once, only $59.8 \%$ reported taking the first dose within the second trimester (19-22weeks) while $39.4 \%$ of women received it during the 3rd trimester (26-30weeks) and rarely $(0.8 \%)$ during the first trimester $(<16$ weeks) (Table 2$)$. The timing of first IPTp dose within the study population as shown in Figure 1 demonstrates that when IPTp was given, it was generally first given from fourth month of gestation and majority of the women received SP between the fifth and sixth months. This is consistent with the WHO guidelines for delivery of IPTp, which recommends the delivery of first IPT dose at each scheduled visit after 'quickening' (16 weeks) to ensure that a high proportion of women received a minimum of two doses of IPT [3]. Furthermore the timing of first IPT dose correlated significantly $(\mathrm{r}=0.919 ; \mathrm{P}<0.001)$ with the timing of first attendance at ANC clinic (Figure 1).

The proportion of women receiving the recommended 2 or 3 doses of IPTp-SP increased $(\mathrm{p}<0.001)$ with the number of ANC visits attended (Table 3). Thus women who received one dose $(\mathrm{OR}=3.7)$ were more likely not to have attended four or more visits (Table 3). More so, there was a significant $(p=0.004)$ association between early first ANC attendance and SP dosage where women who had an early first ANC attendance were more likely $(\mathrm{OR}=0.4 ; 95 \% \mathrm{CI}=0.2-0.7)$ to receive the recommended two or more SP doses. There was no significant association between age, parity, marital status and IPTpSP uptake at an ANC. From 2008-2009, a follow-up study showed an increase in IPTp-SP coverage to $93.5 \%$ (for women who had received at least one dose) and to $64.1 \%$ for those who had received complete IPT (two doses) (Figure 2).

\section{Association between trimester of first ANC visit, SP dosage and malaria parasite infection status at delivery} A significantly higher $(\mathrm{P}<0.001)$ proportion of women with first ANC visit during the third trimester received only one SP dose during pregnancy compared with those who had two or more doses (Table 4). Equally, the prevalence of microscopic malaria parasitaemia was significantly higher $(\mathrm{P}=0.029)$ among parturient women with first clinic visit during the third trimester when compared with those who enrolled in their second trimester of pregnancy (Table 4). Nonetheless the association between trimester of enrolment and malaria parasitaemia at delivery was only significant among women who had one SP dose. Thus microscopic parasitaemia at delivery was significantly higher $\left(\chi^{2}=9.938 ; \mathrm{P}=0.007\right)$ among women who had first ANC attendance in the third trimester and had received one dose $(60.9 \%)$ or no SP $(21.7 \%)$ compared to those who had two or more doses (17.4\%).

\section{Discussion}

This study assessed the determinants of ANC clinic attendance and IPTp-SP uptake among parturient women who seek delivery services at the Mutengene Medical Centre, Cameroon to identify facilitating factors for scaling up IPTp-SP coverage/dosage in the study area. Also 
Table 3 Relationship between ANC visits, individual factors and use of IPTp

\begin{tabular}{|c|c|c|c|c|c|c|c|c|}
\hline \multirow[t]{2}{*}{ Factor } & \multicolumn{2}{|c|}{$\leq 3$ visits } & \multicolumn{2}{|c|}{$\geq 4$ visits } & \multirow{2}{*}{$\begin{array}{c}\text { *Significance level } \\
\left(x^{2} ; p \text {-value }\right)\end{array}$} & \multirow{2}{*}{ \#OR (95\% Cl) } & \multirow{2}{*}{${ }^{\dagger} \mathrm{OR}(95 \% \mathrm{Cl})$} & \multirow{2}{*}{ P-value } \\
\hline & $\%(n)$ & $95 \% \mathrm{Cl}$ & $\%(n)$ & $95 \% \mathrm{Cl}$ & & & & \\
\hline \multicolumn{9}{|l|}{ Age (years) } \\
\hline$\leq 20$ & 40.7 (35) & $30.9-51.3$ & $25.8(51)$ & $20.2-32.3$ & \multirow{3}{*}{$7.670 ; 0.022$} & $2.0(1.2-3.4)$ & $2.0(1.0-4.4)$ & 0.069 \\
\hline $21-25$ & $34.9(30)$ & $25.7-45.4$ & $36.4(72)$ & $30.0-43.2$ & & $0.9(0.6-1.2)$ & $1.5(0.7-3.2)$ & 0.254 \\
\hline$>25$ & $24.4(21)$ & $16.6-34.5$ & $37.9(75)$ & $31.4-44.8$ & & REF & & \\
\hline \multicolumn{9}{|l|}{ Parity } \\
\hline Primiparous & $34.1(30)$ & $25.0-44.4$ & $29.2(58)$ & $23.3-35.8$ & \multirow{3}{*}{$0.765 ; 0.682$} & $1.3(0.7-2.2)$ & $1.2(0.6-2.6)$ & 0.671 \\
\hline Secundiparous & $28.4(25)$ & $20.0-38.6$ & $29.2(58)$ & $23.3-35.8$ & & $1.0(0.6-1.7)$ & $1.4(0.7-2.8)$ & 0.327 \\
\hline Multiparous & $37.5(33)$ & $28.1-47.9$ & $41.7(83)$ & $35.1-48.7$ & & REF & & \\
\hline \multicolumn{9}{|l|}{ Marital status } \\
\hline Unmarried & $35.6(31)$ & $26.4-46.1$ & $20.2(40)$ & $15.2-26.3$ & \multirow[t]{2}{*}{$7.693 ; 0.006$} & $2.2(1.3-3.8)$ & $2.5(1.3-4.6)$ & 0.006 \\
\hline Married & $64.4(56)$ & $53.9-73.6$ & 79.8 (158) & $73.7-84.8$ & & REF & & \\
\hline \multicolumn{9}{|c|}{ Educational level } \\
\hline None & $3.8(3)$ & $1.2-10.5$ & $7.6(14)$ & $4.6-12.3$ & \multirow{4}{*}{$3.740 ; 0.291$} & $0.5(0.1-1.7)$ & $0.8(0.1-5.1)$ & 0.827 \\
\hline Primary & $70.0(56)$ & $59.2-78.9$ & $58.9(109)$ & $51.7-65.8$ & & $1.6(0.9-2.9)$ & $1.4(0.4-5.5)$ & 0.594 \\
\hline Secondary & $21.3(17)$ & $13.7-31.4$ & $29.2(54)$ & $23.1-36.1$ & & $0.7(0.4-1.2)$ & $0.8(0.2-3.3)$ & 0.780 \\
\hline Tertiary & $5.0(4)$ & $2.0-12.0$ & $4.3(8)$ & $2.2-8.3$ & & REF & & \\
\hline \multicolumn{9}{|l|}{ Fever history } \\
\hline Yes & $32.5(26)$ & $23.2-43.4$ & $48.4(93)$ & $41.5-55.5$ & \multirow{2}{*}{$5.829 ; 0.016$} & $0.5(0.3-0.9)$ & $0.5(0.3-0.8)$ & 0.009 \\
\hline No & $67.5(54)$ & $56.6-76.8$ & $51.6(99)$ & $44.5-58.5$ & & REF & & \\
\hline \multicolumn{9}{|c|}{ IPTp-SP dosage } \\
\hline one & $70.3(45)$ & $58.2-80.1$ & $39.1(75)$ & $32.4-46.1$ & \multirow{2}{*}{$18.824 ; 0.001$} & $3.7(2.0-6.8)$ & \multirow[t]{2}{*}{ NA } & \\
\hline Two or more & 29.7 (19) & $19.9-41.8$ & 60.9 (117) & $53.9-67.6$ & & & & \\
\hline
\end{tabular}

${ }^{*}$ Determined by Chi-square (Fischer exact) Test; ${ }^{*}$ determined from Confidence interval calculator, ${ }^{\dagger}$ determined by multinominal logistic regression, OR $=$ Odd ratio, $\mathrm{Cl}=\mathrm{Confidence} \mathrm{interval,} \mathrm{Values} \mathrm{in} \mathrm{bold} \mathrm{indicate}$ significant associations, Significance level $=P<0.05$. 


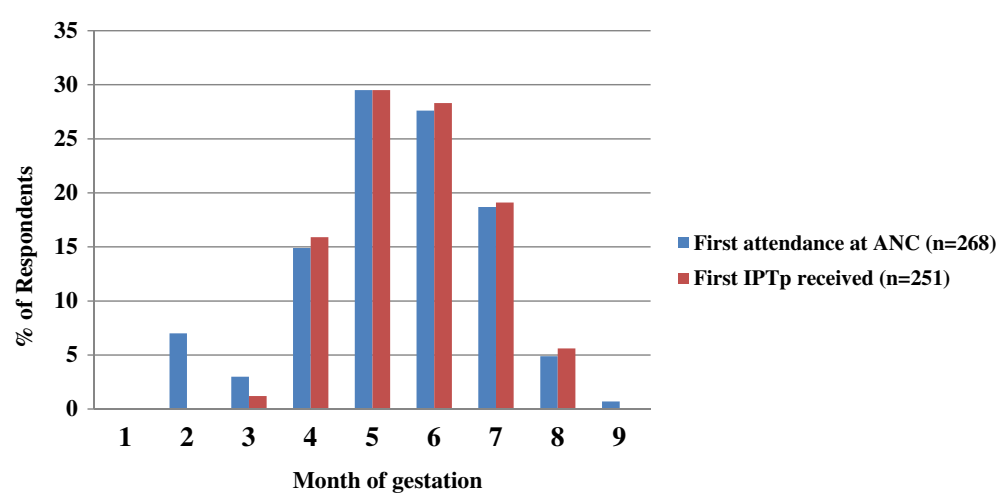

Figure 1 Comparison of timing of first attendance at antenatal clinic with timing of first dose of IPTp in the Mutengene Medical Centre.

the study examined the association between time of first ANC clinic attendance, IPTp-SP dosage and malaria parasitaemia at delivery. The study shows a high coverage of ANC attendance in the Mount Cameroon Area, which ranged from one to twelve visits with a median of five visits. The WHO recommendation of four ANC visits was met by more than $70 \%$ of the interviewed women. These findings indicate a high rate of antenatal care utilization in the study area, similar to that observed in Gabon [17] and Tanzania [21] and offers the potential for implementing the nationally recommended approaches to the prevention and control of malaria [9]. This high attendance can be explained by the fact that Mutengene Medical Centre is the only public health facility that offers antenatal care, preventive, curative and delivery services at affordable costs when compared with the private medical centres in the health area. In Cameroon, in several health centres and public hospitals, health care services have been made affordable for the middle and low-income population since 2007. Intermittent preventive treatment with SP is freely provided to the mothers and is most often available. Evidence from elsewhere has shown that access to services and costs are serious barriers to utilization of ANC services among the underprivileged and thus making such services affordable to the poor is a necessity [22,23]. Although the timing of ANC was also found to be correct with a large proportion of women having their first visit during the second trimester, more than one third (38\%) of women were found to start ANC clinic during the third trimester. Similar findings have been observed in other African countries [24]. A majority of women attended ANC at five months gestation or later and thus represents an opportunity for timely delivery of IPTp-SP. More so, the study showed that the timing of ANC attendance had a significant effect on the uptake of first SP dose. The first SP dose was usually administered after first ANC consultation. In contrast, a previous study in Northeast Tanzania observed that earlier ANC attendance ( $<20$ weeks) had only a limited effect on the uptake of IPTp [25]. Late attendance for some pregnant women (5\%) occurred at or after 32 weeks thus leaving insufficient time for the uptake of a second dose of SP before giving birth. This is in line with recent findings in Benin by d'Almeida et al. [26].

Factors affecting ANC clinic attendance have been reviewed [11]. Women with a fever history were more likely to make an earlier ANC initiation or more visits ( $\geq 4$ visits) in this study suggesting that enrolment and attendance are delayed (or the opposite promoted) depending on the health status of the pregnant woman. A study in Tanzania found that having a history of a previous reproductive loss was a strong predictor for an earlier ANC initiation. Secondly marital status was found to be an independent factor associated with frequent ANC clinic visits rather than the timing of the first visit. Similarly, not being supported by the husband or partner was identified as a factor associated with a later antenatal care enrolment in South-eastern Tanzania [21]. On the contrary, a study in rural Uganda found no significant association between marital status and ANC utilization [12]. Findings by Schatz [27] in Malawi have shown that marriage is an economic necessity for women while studies in Kenya [28] and Ghana [29] noted that husbands have an important economic role that influences women's access to care. More so, some men perceive it as the husband's responsibility to maintain the well-being of his pregnant wife and to ensure that she attends the ANC. Studies have also shown that adolescent pregnant girls are less likely to attend an ANC and seek timely care for malaria [29,30]. There was no significant relationship between age and ANC attendance after adjusting for covariates in exploratory analysis. Other authors have reported no association [21,31].

In 2007 , about $90 \%$ of women received at least one dose but only $53 \%$ received the recommended two dose SP regimen. This observed coverage is in range with findings from other settings. A cross-sectional study in Gabon reported $84.1 \%$ receiving one dose of SP and $57.4 \%$ received 


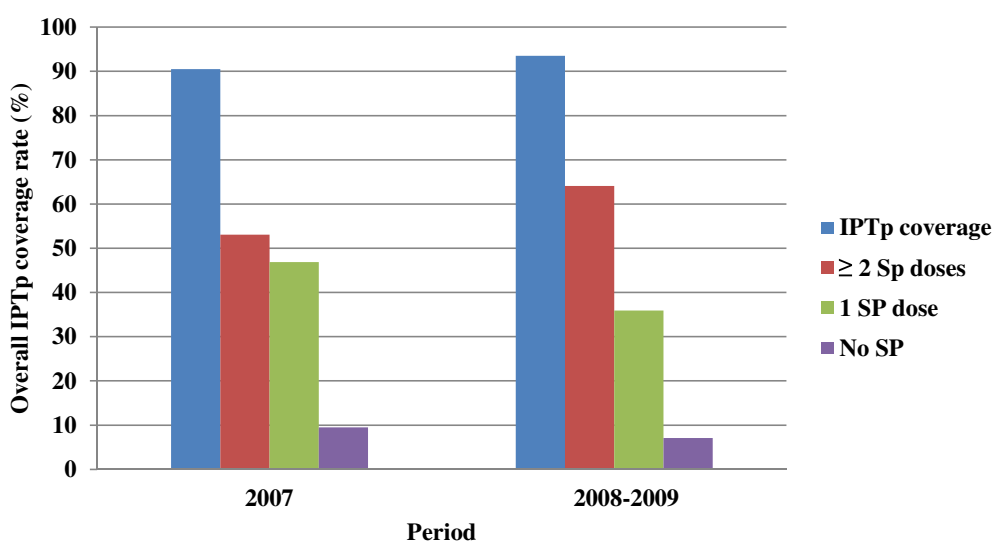

Figure 2 Overall IPTp intake in parturient women in 2007, 2008-2009 in the Mutengene Medical Centre.

at least two doses [17]. In Burkina Faso, 93.5\% took any dose of IPTp/SP and two or more doses of SP were taken by $57.6 \%$ of the women [32]. In 2008-2009, an increase in the coverage rate of IPT-two doses to $64 \%$ was recorded probably due to continuous awareness campaigns organized throughout the country. Similarly, in Benin, the rate of IPT-SP coverage for those who had received complete IPT increased to $68.4 \%$ in 2009 [26]. In the study area, the delivery of the first IPTp-SP dose was timely, generally between 16-24weeks, which is in accordance with the WHO implementation guideline [3]. Firstly, the low coverage of the second SP dose compared to that of first dose in study area could be due to fewer ANC visits ( $\leq 3$ visits). Women who had fewer ANC visits were almost four times likely not to take the recommended SP dosage. This finding confirms reports from Benin and Cameroon where IPT administration varied with the number of prenatal consultations $[26,33]$. Comparable to findings by Bouyou-Akotet et al. [17] in Gabon, it is noteworthy that 39\% of parturient women with more than three ANC visits had partial SP uptake which is a evident that several ANC visits does not necessary ensure complete IPTp uptake, thus identification of facilitating factors to enhance uptake of complete IPTp dose warrants investigation. Secondly, achievement of full SP dose was also prevented by late enrolment at the ANC, frequently delayed until the third trimester and at or after 32 weeks of gestation for certain women, in line with reports from Benin [26]. This study further shows that women who attended first ANC clinic in the third trimester and had one or no SP dose frequently had microscopic parasitaemia at delivery suggesting that late ANC may have important implications in the prevalence of malaria parasitaemia in women at delivery.

\section{Conclusion}

Marital status and fever history independently influenced ANC clinic attendance while uptake of one SP dose was associated with late first ANC visit and fewer clinic visits. Thus earlier ANC clinic attendance may improve coverage of two or more SP doses in the study area. Given that about $40 \%$ of parturient women who had recommended number ANC visits ( $\geq 4$ visits) had partial SP uptake suggests that other factors besides number and timing of ANC clinic visits affect the delivery, access, and use of interventions to prevent malaria in pregnancy in the study area. Thus identification of barriers to the

Table 4 Association of trimester of first ANC visit with SP dosage and malaria infection status at delivery

\begin{tabular}{|c|c|c|c|c|}
\hline Factor/Trimester & First $\%(n)$ & Second \% (n) & Third \% (n) & *Significance level \\
\hline \multicolumn{5}{|l|}{ SP dosage } \\
\hline Two or more & $33.3(2)$ & $63.5(101)$ & $28(28)$ & \multirow[t]{3}{*}{$x^{2}=35.361 ; p<0.001$} \\
\hline One & $50(3)$ & $34.6(55)$ & $62(62)$ & \\
\hline No SP & $16.7(1)$ & $1.9(3)$ & $10(10)$ & \\
\hline \multicolumn{5}{|c|}{ Malaria infection status } \\
\hline Microscopic ${ }^{\&}$ & $0(0)$ & $18.8(30)$ & $22.5(23)$ & \multirow[t]{3}{*}{$x^{2}=10.795 ; \mathrm{p}=0.029$} \\
\hline Submicroscopic ${ }^{\#}$ & $66.7(4)$ & $17.5(28)$ & $15.7(16)$ & \\
\hline Negative & $33.3(2)$ & $63.8(102)$ & $61.8(63)$ & \\
\hline
\end{tabular}

*Determined by Pearson Chi-square Test; Significance level $=\mathrm{P}<0.05$.

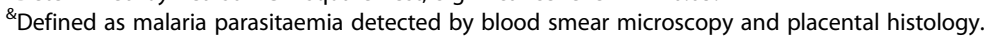

"Defined as malaria parasitaemia detected by placental histology only. 
provision and uptake of IPTp warrants further investigation. More so, educating pregnant women on focused ANC clinic visits is recommended.

\section{Competing interests}

The authors declare that they have no competing interests.

\section{Authors' contributions}

JKAK: Conception and design of study, data collection, analysis, interpretation and manuscript preparation. EAA: Supervision and critically reading of manuscript for important and intellectual content. JKAK TOA, RNM, HFC, RBT: Data collection on IPTp during the 2008-2009 survey. NB and JMNM: Contributed in placental histopathological studies. ESE: Design of study and critically reading of manuscript for important and intellectual content; MTB: Supervision and revision of manuscript. All authors read and approved the manuscript.

\section{Acknowledgements}

We thank the entire staff of the Mutengene Medical Centre and the mothers who volunteered to participate in this study. We are grateful to Mr Epie and all the midwives in particular for cooperation and support in this research work. Our profound gratitude to the Jean-Baptist Bengono and the entire staff of the Anatomy and Pathology Laboratory (ANAPATH), University of Yaoundé teaching hospital (CHU), Cameroon and the Department of Immunology, University of Stockholm, Sweden for their technical assistance. This investigation received financial support from MIM/TDR/WHO Malaria research project [Project A60040 to A.E.A] and Swedish Institute, Stockholm [Guest Scholarship to A. J. K.].

\section{Author details}

${ }^{1}$ Department of Zoology and Animal Physiology, University of Buea, Buea 63, Cameroon. ${ }^{2}$ Department of Biochemistry and Molecular Biology, University of Buea, Buea 63, Cameroon. ${ }^{3}$ Department of Microbiology and Parasitology, University of Buea, Buea 63, Cameroon. ${ }^{4}$ Department of Anatomy and Pathology, University of Yaoundé Teaching Hospital, Yaoundé 63, Cameroon. ${ }^{5}$ Department of Molecular Bioscience, Wenner-Gren Institute, Stockholm University, SE- 10691 Stockholm, Sweden.

Received: 5 December 2013 Accepted: 17 April 2014 Published: 30 April 2014

\section{References}

1. Standley GC, Lewis TLT, Pinker G: Antenatal care. In: Obstetrics by Ten teachers. London: Butler \& Tanner Ltd; 1985.

2. CEMACH: Saving mothers lives: reviewing maternal deaths to make mothers safer - 2003-2005. The Seventh Report of the Confidential Enquiry into Maternal and Child Health in the United Kingdom. London: RCOG Press; 2007.

3. Steketee RW, Nahlen BL, Parise ME, Menendez C: The burden of malaria in pregnancy in malaria-endemic countries. Am J Trop Med Hyg 2001, 6:28-35.

4. WHO: A Strategic Framework for malaria prevention and control during pregnancy in the African Region. Brazzaville: World Health Organization Regional Office for Africa; 2004. AFR/MAL/04/01.

5. Menéndez C, D'Alessandro U, ter Kuile FO: Reducing the burden of malaria in pregnancy by preventive strategies. Lancet Infect Dis 2007, 7:126-135.

6. Mbacham WF, Evehe MSB, Netongo PM, Ateh IA, Mimche PN, Ajua A, Nji AM, Domkam I, Echouffo-Tcheuqui JB, Tawe B, Hallett R, Roper C, Targett G, Greenwood B: Efficacy of amodiaquine, sulphadoxine-pyrimethamine and their combination for the treatment of uncomplicated Plasmodium falciparum malaria in children in Cameroon at the time of policy change to artemisinin-based combination therapy. Malar J 2010, 9:34.

7. Shulman CE, Dorman EK, Cutts F, Kawuondo K, Bulmer JN, Peshu N, Marsh $\mathrm{K}$ : Intermittent sulphadoxine-pyrimethamine to prevent severe anaemia secondary to malaria in pregnancy: a randomised placebo-controlled trial. Lancet 1999, 353:632-636.

8. Challis K, Osman NB, Cotiro M, Nordahl G, Dgedge M, Bergstrom S: Impact of a double dose of sulphadoxine-pyrimethamine to reduce prevalence of pregnancy malaria in southern Mozambique. Trop Med Int Health 2004, 9:106-1073.

9. WHO: Malaria in Pregnancy: Guidelines for Measuring Key Monitoring and Evaluation Indicators. Geneva: World Health Organization; 2007.
10. WHO: World malaria report; 2010.

11. Hill J, Hoyt J, van Eijk AM, D'Mello-Guyett L, ter Kuile FO, Steketee R, Smith H, Webster J: Factors affecting the delivery, access, and use of interventions to prevent malaria in pregnancy in sub-Saharan Africa: a systematic review and meta- analysis. PLOS Med 2013, 10:e10011488.

12. Ndyomugyenyi $R$, Neema $S$, Magnussen $P$ : The use of formal and informal services for antenatal care and malaria treatment in rural Uganda. Health Policy Plan 1998, 13:94-102.

13. Mubyazi G, Bloch P, Kamugisha M, Kitua A, ljumba J: Intermittent preventive treatment of malaria during pregnancy: a qualitative study of knowledge, attitudes and practices of district health managers, antenatal care staff and pregnant women in Korogwe District. North-Eastern Tanzania. Malar J 2005, 4:31.

14. Holtz TH, Kachur SP, Roberts JM, Marum LH, Mkandala C, Chizani N, Macheso A, Parise ME: Use of antenatal care services and intermittent preventive treatment for malaria among pregnant women in Blantyre District, Malawi. Trop Med Int Health 2004, 9:77-82.

15. Kiwuwa MS, Mufubenga P: Use of antenatal care, maternity services, intermittent presumptive treatment and insecticide treated bed nets by pregnant women in Luwero district Uganda. Malar J 2008, 7:44.

16. Victora CG, Matijasevich A, Silveira MF, Santos IS, Barros AJD, Barros FC: Socio-economic and ethnic group inequities in antenatal care quality in the public and private sector in Brazil. Health Policy Plan 2010, 25:253-261.

17. Bouyou-Akotet MK, Mawili-Mboumba DP, Kombila M: Antenatal care visit attendance, intermittent preventive treatment and bed net use during pregnancy in Gabon. BMC Pregnancy Childbirth 2013, 13:52.

18. Anchang-Kimbi JK, Achidi EA, Nkegoum B, Sverremark-Ekstrom E, Troye-Blomberg M: Diagnostic comparison of malaria infection in peripheral blood, placental blood and placental biopsies in Cameroonian parturient women. Malar J 2009, 8:126.

19. Achidi EA, Anchang JK, Minang TJ, Boyo MA, Sinju CM, Troye-Blomberg M, Mokube JA: Malaria infection in pregnancy and its effect on haemoglobin levels in women from a malaria endemic area of Fako Division, South West Province, Cameroon. J Obstetr Gynaecol 2005, 25:235-240.

20. Achidi EA: Malaria baseline studies towards characterising and establishing a clinical trial site at Mutengene, South West Region Cameroon. http://www.edctp.org/uploads/tx_viprojects/Eric_AchidiProject_Profile_-_SF_40200_Eric_Achidi.pdf 2012

21. Gross K, Alba S, Glass TR, Schellenberg JA, Obrist B: Timing of antenatal care for adolescent and adult pregnant women in South-Eastern Tanzania. BMC Pregnancy Childbirth 2012, 12:16.

22. Fotso JC, Ezeh A, Oronje R: Provision and use of maternal health services among urban poor women in Kenya: What do we know and what can we do? J Urban Health 2008, 85:428-442.

23. Onah HE, Ikeako LC, lloabachie GC: Factors associated with the use of maternity services in Enugu, southeastern Nigeria. Soc Sci Med 2006, 63:1870-1878

24. Hill J, Kazembe P: Reaching the Abuja target for intermittent preventive treatment of malaria in pregnancy in African women: a review of progress and operational challenges. Trop Med Int Health 2006, 11:409-418.

25. Anders K, Marchant T, Chambo P, Mapunda P, Reyburn H: Timing of intermittent preventive treatment for malaria during pregnancy and the implications of current policy on early uptake in North-East Tanzania. Malar J 2008, 7:79.

26. d'Almeida TCDA, Agboton-Zoumenou MA, Garcia A, Massougbodji A, Briand $V$, Imorou $Y$, Cottrell G: Evaluation Field of the intermittent preventive treatment of malaria during pregnancy (IPTp) in Benin: evolution of the coverage rate since its implementation. Parasit Vectors 2011, 4:108.

27. Schatz E: "Take your mat and go!": Rural Malawian women's strategies in the HIV/AIDS era. Cult Health Sex 2005, 7:479-492.

28. Molyneux CS, Murira G, Masha J, Snow RW: Intra-household relations and treatment decision-making for childhood illness: a Kenyan case study. J Biosocial Sci 2002, 34:109-131.

29. Tolhurst R, Nyonator FK: Looking within the household: gender roles and responses to malaria in Ghana. Trans R Soc Trop Med Hyg 2006, 100:299-304.

30. Mbonye AK, Neema S, Magnussen P: Preventing malaria in pregnancy: a study of perceptions and policy implications in Mukono district, Uganda. Health Policy Plan 2006, 21:17-26.

31. Babalola S, Fatusi A: Determinants of use of maternal health services in Nigeria - looking beyond individual and household factors. BMC Pregnancy Childbirth 2009, 9:43. 
32. Sirima SB, Cotte AH, Konate A, Moran AC, Asamoa K, Bougouma EC, Diarra A, Ouedraogo A, Parise ME, Newman RD: Malaria prevention during pregnancy: assessing the disease burden one year after implementing a program of intermittent preventive treatment in Koupela District, Burkina Faso. Am J Trop Med Hyg 2006, 75:205-211.

33. Tonga C, Kimbi HK, Anchang-Kimbi JK, Nyabeyeu HN, Bissemou ZB, Lehman LG: Malaria risk factors in women on intermittent preventive treatment at delivery and their effects on pregnancy outcome in Sanaga-Maritime, Cameroon. PLOS ONE 2013, 8:e65876.

doi:10.1186/1475-2875-13-162

Cite this article as: Anchang-Kimbi et al:: Antenatal care visit attendance, intermittent preventive treatment during pregnancy (IPTp) and malaria parasitaemia at delivery. Malaria Journal 2014 13:162.

\section{Submit your next manuscript to BioMed Central and take full advantage of:}

- Convenient online submission

- Thorough peer review

- No space constraints or color figure charges

- Immediate publication on acceptance

- Inclusion in PubMed, CAS, Scopus and Google Scholar

- Research which is freely available for redistribution 